\title{
The Mechanism of Embolic Watershed Infarction: Experimental Studies
}

\author{
M.S. Pollanen and J.H.N. Deck
}

\begin{abstract}
The mechanism for the preferential distribution of emboli to cerebral arterial borderzone regions, known to cause some watershed infarcts was studied. We hypothesized that emboli of a specific size range are selectively directed to the arterial borderzones due to the tendency of emboli to bypass the small arterial branches which emerge proximal to major borderzones. To test this hypothesis we perfused the brains of cadavers with suspensions of 90-210 $\mu \mathrm{m}$ glass microspheres and chemically extracted the particles from various arterial territories and a watershed zone. Particles in the $150-210 \mu \mathrm{m}$ size range were found to be preferentially distributed to the watershed zone whereas particles less than $150 \mu \mathrm{m}$ in size were randomly dispersed in leptomeningeal arteries of all vascular regions. To assess the role of branch size on the concentration of emboli at bifurcations, we perfused artificial analogs of evenly and unevenly branching bifurcations with suspensions of $90-150 \mu \mathrm{m}$ and $150-210 \mu \mathrm{m}$ particles. Branching cylinders with symmetrical branches contained the same concentration of particles, independent of particle size. In contrast, when one branch was one-quarter the size of the other, the concentration of $150-210 \mu \mathrm{m}$ particles in the asymmetric branch was approximately $65 \%$ of the main trunk. Particles $90-150 \mu \mathrm{m}$ in size were evenly distributed despite variation in branch size. These results indicate that emboli, of a limited range of size, may be selectively propagated to the distal ramifications of subarachnoid arteries located in the watershed zone rather than diverging into small calibre branches which arise along the way.
\end{abstract}

\begin{abstract}
RÉSUMÉ: Études expérimentales du mécanisme de l'infarctus embolique des zones de partage du lit vasculaire Le mécanisme de la distribution préférentielle des embolies aux régions limites du réseau artériel cérébral, connues comme causant des infarcissements des zones de partage du lit vasculaire, a été étudié. Nous avons émis l'hypothèse que les embolies de certaines tailles spécifiques sont dirigées sélectivement aux zones artérielles limites à cause de la tendance des embolies à éviter les petites branches artérielles qui émergent à proximité des zones frontières importantes. Afin de vérifier cette hypothèse, nous avons perfusé le cerveau de cadavres avec une suspension de microsphères de verre de 90-210 $\mu \mathrm{m}$ et nous avons extrait chimiquement les particules de différents territoires artériels et d'une zone de partage du lit vasculaire. Les particules de la taille 150-210 $\mu \mathrm{m}$ ont été retrouvées préférentiellement distribuées à la zone de partage du lit vasculaire alors que les particules plus petites que $150 \mu \mathrm{m}$ étaient dispersées de façon aléatoire dans les artères leptoméningées de toutes les régions vasculaires. Pour évaluer le rôle de la taille des branches artérielles sur la concentration des embolies aux bifurcations, nous avons perfusé des analogues artificiels à bifurcations symétriques et asymétriques avec des suspensions de particules de $90-150 \mu \mathrm{m}$ et de 150-210 $\mu \mathrm{m}$. Les cylindres à branches symétriques contenaient la même concentration de particules, indépendamment de la taille des particules. Par contre, quand une branche était le quart de la taille de l'autre, la concentration de particules de 150 $210 \mu \mathrm{m}$ dans la branche asymétrique était approximativement $65 \%$ de celle du tronc principal. Les particules de 90 $150 \mu \mathrm{m}$ étaient distribuées également en dépit de variations dans la taille des branches. Ces résultats indiquent que les embolies, d'une certaine gamme de tailles, peuvent sélectivement gagner les ramifications distales des artères sousarachnö̈diennes situées dans la zone de partage du lit vasculaire plutôt que d'être déviées vers les branches de petit calibre qui prennent naissance sur leur trajet.
\end{abstract}

Can. J. Neurol. Sci. 1990; 17: 395-398

Cerebral borderzone or watershed infarcts (WI) are ischemic lesions which occur along the boundary zone between major cerebral arterial territories. The majority of WI are caused by diminished perfusion of distal arteries ${ }^{1,2}$ usually due to systemic hypotension ${ }^{3}$ or stenosing carotid atherosclerosis. ${ }^{4}$ However, there are some cases of WI that are not explained by cerebral hypoperfusion but are seemingly the result of vascular occlusion by thromboemboli, 5.6 cholesterol emboli, 5.7 .8 .9 or tumour emboli. 5

The underlying mechanism for embolic WI is not known. However, two distinct mechanisms to account for the selective distribution of emboli have been proposed emphasizing arterial

From the Department of Pathology, Division of Neuropathology, Toronto General Hospital, 200 Elizabeth Street, EC-4-316, Toronto. Ontario Received December 28, 1989. Accepted in final form May 29, 1990

Presented at the Canadian Association of Neuropathologists annual meeting in Montreal, Quebec, September, 1989, with honourable mention awarded for Mary Tom Award

Reprint requests to: Dr. J.H.N. Deck, Department of Pathology, Division of Neuropathology, EC-4-316, Toronto General Hospital, 200 Elizabeth Street, Toronto, Ontario, Canada M5G 2C4 
branch angle 5 and arterial branch diameter. 6 The latter mechanism suggests that emboli, of a limited range of size (approximately $200 \mu \mathrm{m}$ ), travel to the distal branches of cerebral arteries located in the watershed zones, rather than diverging into arterial branches of smaller diameter, due to the tendency of the emboli to remain in the centre of laminar flow. 10.11 Accordingly, cerebral microemboli are almost exclusively concentrated in borderzone-bound arteries and when vessel diameters diminish in the borderzone region, emboli become arrested causing infarction. For the first time we report experimental validation of this explanation by showing (1) preferential size specific distribution of particles to watershed zones in a cadaver, and (2) size dependent distribution of suspended particles in unevenly branching cylinders as models of arterial bifurcations.

\section{Materials ANd Methods}

\section{Cadaveric Model}

In three cadavers ( 12 hours post mortem) with minimal atherosclerosis and no neurologic history, the internal carotid arteries in the neck were isolated and the right common carotid artery ligated. The left internal carotid artery was cannulated, the cannula attached to a perfusion apparatus and the cerebral circulation flushed with physiologic saline under near systolic constant pressure $(300 \mathrm{~mL})$ until effluent from the jugular veins was clear. The perfusion apparatus was then filled with perfusate containing anticoagulated whole blood with a suspension of $90-120 \mu \mathrm{m}$ glass microspheres (Glass Microcarrier Beads, Sigma, St. Louis, MO) in a total volume of $300 \mathrm{~mL}$. The left cerebral hemisphere was then perfused with $1.3 \times 10^{6}$ microspheres under constant pressure and a flow rate $(10 \mathrm{~mL} / \mathrm{s})$. The skull was then opened, the brain removed and one gram of leptomeningeal arteries were stripped from each of the left anterior and middle cerebral arterial territories and the anterior-middle cerebral arterial watershed zone. Collected arteries were solubilized in $35 \%$ nitric acid $\left(37^{\circ} \mathrm{C}, 12\right.$ hours) and microspheres extracted by centrifugation ( $200 \mathrm{~g}, 2$ minutes). Microspheres were enumerated with a light microscope, and size was determined using a calibrated ocular graticule. Values are expressed as per cent particles recovered per vascular region from one representative cadaveric perfusion.

\section{In Vitro Model}

Artificial metal bifurcations were made as analogues to cerebral arterial bifurcations, similar to previous work." Isolated segments were constructed with a constant trunk diameter of $4 \mathrm{~mm}$ and emergent branches either $1 \mathrm{~mm}$ to $4 \mathrm{~mm}$ in cross sectional diameter. The branch angle was constant at 60 degrees. Individual segments were perfused with known concentrations of particles (800-1000 particles/mL in $13 \%$ Glycerine to match viscosity of blood) in the $90-150 \mu \mathrm{m}$ size range and in other experiments using particles $150-210 \mu \mathrm{m}$ in size. Segments were perfused at a constant flow rate of $10 \mathrm{~mL} / \mathrm{s}$, using a peristaltic pump to generate pulsatile flow, and the effluent from the various size branches and the trunk were collected. The concentration of particles from the even and uneven branches were determined by microscopic enumeration of $150 \mu \mathrm{L}$ aliquots. Results are expressed as number of particles $/ \mathrm{mL}$ in the respective branches. The reported values are means and standard deviations of three separate perfusion experiments.

\section{Results}

\section{Cadaveric Model}

Microspheres in the $90-150 \mu \mathrm{m}$ size range were found to be randomly dispersed in leptomeningeal arteries of the various territories (Figure 1a). However, microspheres in the 150-210 $\mu \mathrm{m}$ size range were preferentially distributed to the watershed zone (Figure lb).

A.

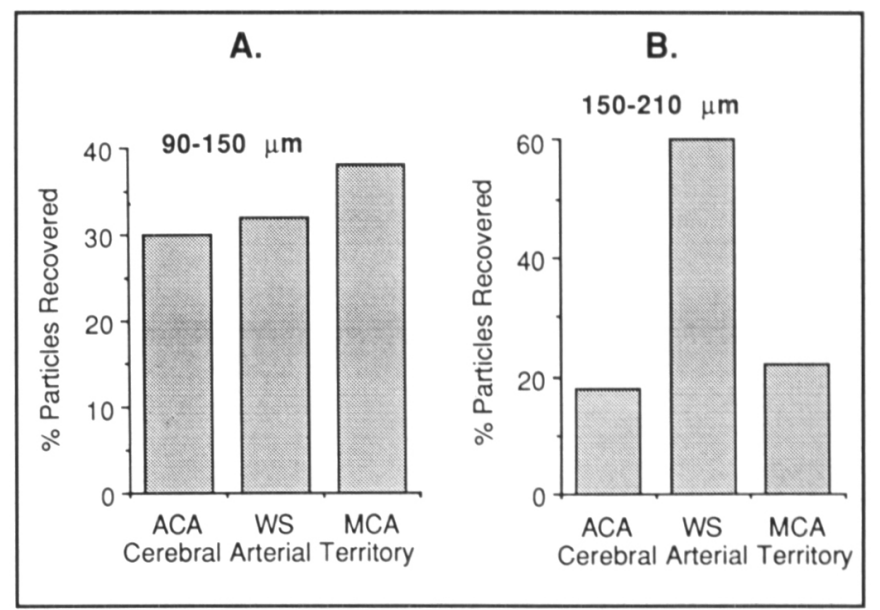

Figure $I-(A)$ Relative percentages of $90-150 \mu \mathrm{m}$ particles recovered fiom leptomeningeal arteries fiom the anterior cerebral (ACA), middle cerebral (MCA) and watershed (WS) territories. Particles in the $90-150 \mu \mathrm{m}$ are evelly distributed to all vascular regions. (B) - Particles in the 150-210 $\mathrm{\mu m}$ are preferentially distributed to the watershed (WS) zone.

\section{In Vitro Model}

Particles in the $90-150 \mu \mathrm{m}$ size range were evenly distributed to both branches at the bifurcations independent of the size of the branches (Figure 2). When particles in the $150-210 \mu \mathrm{m}$ range were perfused through evenly branching bifurcations, the concentration of particles was similar in both branches (Figure $3 a)$. However, when particles in the $150-210 \mu \mathrm{m}$ range were perfused into the unevenly branching bifurcations, the asymmet-

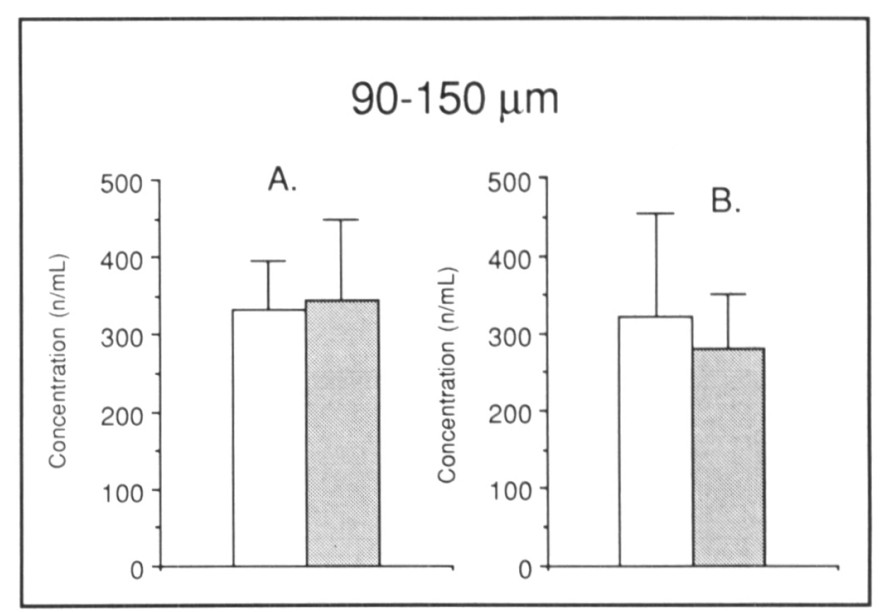

Figure $2-$ (A) Bifurcations with two branches of the same size (shaded and clear bars) perfused with 90-150 $\mathrm{mm}$ particles showing even distribution of particles in both branches. $(B)$ Bifurcations with one branch one-quarter the size (shaded) of the other (clear) show even distribution of $90-150 \mu \mathrm{m}$ particles. 


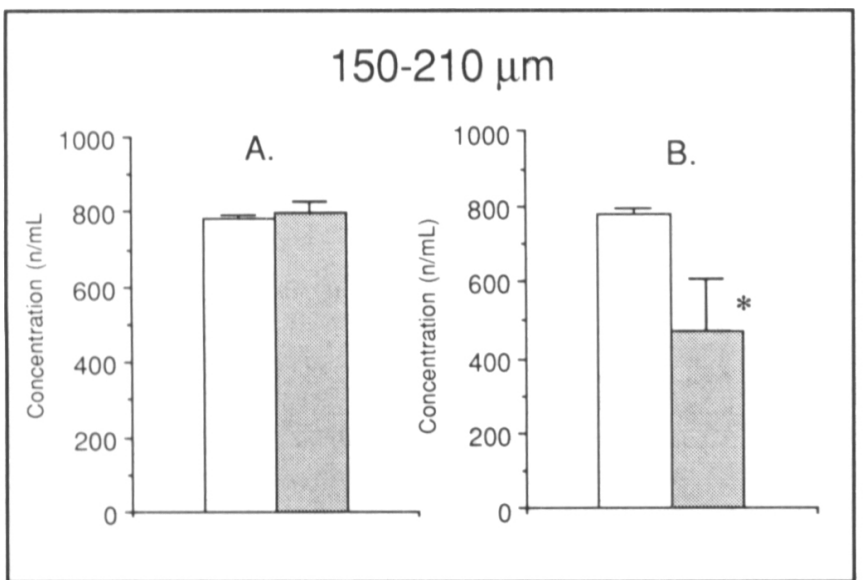

Figure $3-$ (A) Bifurcations with two branches of the same size (shaded and clear), perfused with $150-210 \mu \mathrm{m}$ particles show even distribution in both branches. $(B)$ Bifurcations with one branch one-quarter the size (shaded) of the other (clear) contain fewer $150-210 \mu \mathrm{m}$ particles/ $m L$. (*indicates $p \leq 0.05$ by ANOVA)

ric branch (one-quarter of the parent trunk) was found to have $65 \%$ of the concentration of particles in the main trunk. Decreasing the branch diameter produced a relative concentration drop in particles contained in the asymmetric branch.

\section{Discussion}

Infarcts along the boundary regions of major cerebral arterial distributions, known as watershed infarcted (WI) are usually correlated with either cerebral hypoperfusion or embolic occlusion of distal arterial segments in the brain. ${ }^{4}$ Studies using the hypotensive-primate model reproduce the topography of infarction found in human cases of WI with presumed cerebral hypoperfusion. 1,3 However, no experimental model has reproduced selective embolization to watershed zones. 1,2

In the present experiments, microspheres $150-210 \mu \mathrm{m}$ in diameter were selectively distributed to watershed zones in cadavers, when suspended in whole blood, rather than being randomly dispersed. In addition, as previously reported in an animal model, artificial emboli in the $90-150 \mu \mathrm{m}$ range were non-selective in arterial distribution throughout the brain 1.2 indicating that preferential sorting of emboli occurs for particles of a limited size range. This is consistent with previous observations which indicate that emboli overlying WI are seldom less than $200 \mu \mathrm{m}$ in size, and very rarely less than $100 \mu \mathrm{m}$ in size. ${ }^{9}$

Two mechanisms have been proposed to explain the preferential distribution of emboli in the cerebral circulation. The first, proposed by Torvik and Skullerud, 5 emphasized the sharp angulation of branching vessels, suggesting that emboli are unlikely to enter such branches. However, in vitro flow studies indicate that branch angle is likely less important to the fluid dynamics in arterial branches compared to the asymmetric diameter of the branch vessel as compared to the parent vessel. ${ }^{13}$ On this basis, we emphasized an alternative property of the cerebral circulation to account for selective watershed embolism, ${ }^{6}$ suggesting that particulate emboli in arterial flow are more likely to remain in a centralized stream and not diverge into asymmetric arterial branches characteristic of cerebral arteries. In support of the latter mechanism, in vitro flow models indicate that 1) particles flowing in cylindrical tubes tend to aggregate in a central stream, ${ }^{14}$ 2) fluid which enters asymmetric cylindrical branches is derived from the peripheral stream of the parent vessel rather than the central stream ${ }^{10.15}$ and 3 ) experimental studies of suspended particles in laminar flow are subjected to "phase separation" where particles tend not to diverge into branches emerging from main trunks."

Our experimental system using isolated artificial bifurcations reproduces the phase separation effect indicating that arterial branches with small diameters relative to parent arteries may contain fewer emboli than evenly branching arteries in vivo. Therefore, in the case of embolic WI, if emboli remain in the central stream of blood in major subarachnoid arteries rather than entering asymmetric branches, the majority of emboli become watershed bound and arrest only when the arterial lumen tapers to the size of the emboli, in the most distal arterial segments.

Experimental studies show that phase separation occurs only with specific ratios of emboli diameter to artery diameter. "One possibility which can be inferred from the in vitro flow studies is that particles of greater size are more centrally located in arterial flow when suspended in a medium with relatively smaller sized particles. In this way, under natural conditions and in the cadaveric and in vitro systems reported here, $90-150 \mu \mathrm{m}$ emboli would diverge into asymmetric arterial branches and not be specifically localized to watershed zones.

In summary, the selective distribution of emboli in the cerebral circulation previously reported to cause WI has been reproduced in a cadaveric model. Using a perfusion system of isolated arteries, uneven arterial branches tend to contain less emboli than even branches suggesting that arterial branch diameter is important to stream sorting of emboli in watershed bound arteries.

\section{ACKNOWLEDGEMENT}

The authors wish to thank Dr. H.J. Robinson for helpful discussions.

\section{REFERENCES}

1. Brierley JB, Excell BJ. The effect of profound hypotension upon the brain of M. Rhesus. Physiological and pathological observations. Brain 1966; 89: 269-298.

2. Brierley JB, Prior PF, Calverley J, et al. The pathogenesis of neuronal damage along the cerebral arterial boundary zones in Papio Anubis. Brain 1980; 103: 929-965.

3. Adams JH, Brierley BJ, Connor RCR, et al. The effects of systemic hypotension upon the human brain. Clinical and neuropathologic observations in 11 cases. Brain 1966; 89: 235-268.

4. Torvik A. The pathogenesis of watershed infarcts in the brain. Stroke 1984; 15: 221-223.

5. Torvik A, Skullerud K. Watershed infarcts in the brain caused by microemboli. Clin Neuropathol 1982; 1: 99-105.

6. Pollanen MS, Deck, JHN. Directed embolization is an alternate cause of cerebral watershed infarction. Arch Pathol Lab Med 1989; 113: 1139-1141.

7. Beal MF, Williams RS, Richardson EP, et al. Cholesterol embolism as cause of transient ischemic attacks and cerebral infarction. Neurology 1981; 31: 860-865.

8. McKinnon DW, Bulkley BH, Green WR, et al. Fatal cerebral atheromatous embolization after cardiopulmonary bypass. J Thorac Cardiovas Surg 1976; 71: 741-745.

9. Price DL, Harris J. Cholesterol emboli in cerebral arteries as a complication of retrograde aortic perfusion during cardiac surgery. Neurology 1970; 20: 1209-1214. 
10. Charm SE, Kurland GS. Flow behaviour of blood. In: Blood Flow and Microcirculation. New York: John Wiley and Son 1974; 87-97.

11. Bugliarello G, Hsiao GCC. Phase separation in suspensions flowing through bifurcations: a simplified hemodynamic model. Science $1964 ; 143: 469-47$ I.

12. Steegman AT, DeLaFuente J. Experimental cerebral embolism II. Microembolism of rabbit brain with seran polymer resin. J Neuropathol Exp Neurol 1959; 18: 537-558.

13. Pedley TJ. Flow in asymmetric bifurcations. In: The Fluid Mechanics of Large Blood Vessels. Cambridge, USA: Cambridge University Press 1980; 260-261.
14. Charm SE, Kurland GS, Brown SL. The flow characteristics of blood suspensions. In: Biomedical Fluid Mechanics Symposium. New York: The American Society of Mechanical Engineers 1966; 89-93.

15. Segre G, Silberger A. Behaviour of macroscopic rigid spheres in Pouiseuelle flow. Part 1. Determination of local concentration by statistical analysis of particle passage through crossed light beams. J Fluid Mech 1962; 14: 115-136. 